\title{
Association Between Gastroesophageal Reflux Disease After Pneumatic Balloon Dilatation and Clinical Course in Patients With Achalasia
}

\author{
Yang Won Min, Jin Hee Lee, Byung-Hoon Min, Jun Haeng Lee, Jae J Kim and Poong-Lyul Rhee* \\ Department of Medicine, Samsung Medical Center, Sungkyunkwan University School of Medicine, Seoul, Korea
}

\section{Background/Aims}

The occurrence of gastroesophageal reflux disease (GERD) is known to be associated with lower post-treatment lower esophageal sphincter pressure in patients with achalasia. This study aimed to elucidate whether GERD after pneumatic balloon dilatation (PD) has a prognostic role and to investigate how the clinical course of GERD is.

\section{Methods}

A total of 79 consecutive patients who were first diagnosed with primary achalasia and underwent PD as an initial treatment were included in this retrospective study. Single PD was performed using a $3.0 \mathrm{~cm}$ balloon. The patients were divided into two groups: 1) who developed GERD after PD (GERD group) and 2) who did not develop GERD after PD (non-GERD group). GERD was defined as pathological acid exposure, reflux esophagitis or typical reflux symptoms.

\section{Results}

Twenty one patients (26.6\%) developed GERD after PD during follow-up. There were no significant differences between the two groups in demographic or clinical factors including pre- and post-treatment manometric results. All patients in GERD group were well responsive to maintenance proton pump inhibitor therapy including on demand therapy or did not require maintenance. During a median follow-up of 17.8 months (interquartile range, 7.1-42.7 months), achalasia recurred in 15 patients (19.0\%). However, the incidence of recurrence did not differ according to the occurrence of GERD after PD.

\section{Conclusions}

GERD often occurs after even a single PD for achalasia. However, GERD after PD is well responsive to PPI therapy. Our data suggest that GERD after PD during follow-up does not appear to have a prognostic role.

\section{(J Neurogastroenterol Motil 2014;20:212-218)}

\section{Key Words}

Esophageal achalasia; Gastroesophageal reflux; Pneumatic balloon dilatation; Prognosis

Received: October 18, 2013 Revised: January 5, 2014 Accepted: January 19, 2014

(C) This is an Open Access article distributed under the terms of the Creative Commons Attribution Non-Commercial License (http://creativecommons. org/licenses/by-nc/3.0) which permits unrestricted non-commercial use, distribution, and reproduction in any medium, provided the original work is properly cited.

*Correspondence: Poong-Lyul Rhee, MD, PhD

Department of Medicine, Sungkyunkwan University School of Medicine, Samsung Medical Center, 81 Irwon-ro, Gangnam-gu, Seoul 135-710, Korea

Financial support: None.

Tel: +82-2-3410-3409, Fax: +82-2-3410-6983, E-mail: plrhee@skku.edu

Conflicts of interest: None.

Author contributions: Yang Won Min contributed to data analysis and interpretation and drafted the manuscript; Jin Hee Lee contributed to data analysis and interpretation; Byung-Hoon Min, Jun Haeng Lee and Jae J Kim performed critical revision of the manuscript; Poong-Lyul Rhee designed and coordinated the study, contributed to data interpretation and edited the manuscript.

ORCID: Yang Won Min, http://orcid.org/0000-0001-7471-1305; Jae J Kim, http://orcid.org/0000-0002-0226-1330. 


\section{Introduction}

Achalasia is a primary esophageal motor disorder of unknown etiology in which there is degeneration of neurons in the wall of the esophagus leading to absence of peristalsis and impaired relaxation of the lower esophageal sphincter (LES). ${ }^{1,2}$ The symptoms of achalasia are dysphagia for solids and liquids, regurgitation of undigested food, respiratory symptoms (nocturnal cough, recurrent aspiration and pneumonia), chest pain and weight loss. ${ }^{3,4}$

Achalasia is not curable, which leads to be a chronic condition. Currently pneumatic balloon dilatation (PD), surgical myotomy, per-oral endoscopic myotomy (POEM) and botulinum toxin injection have been performed as initial treatment for achalasia depending on patient's condition and center expertise. ${ }^{4-7}$ All these treatment options aimed at reducing the elevated pressure of LES. ${ }^{1,8-10}$ However, the LES hypertonicity returns over time and repeated interventions are needed. Gastroesophageal reflux disease (GERD) may occur due to disrupted LES after PD as well as after surgery and POEM. ${ }^{11-14}$ Then, the occurrence of GERD after treatment might be a prognostic factor for a favorable long-term outcome in patients with achalasia.

This study aimed to determine whether GERD after PD has a prognostic role for recurrence free survival (RFS) in patients who received PD for achalasia and to investigate how often GERD occurs in achalasia patients who undergo PD and how the clinical course of GERD after PD is.

\section{Materials and Methods}

\section{Patients}

This retrospective study included data from a total of 82 consecutive patients who were diagnosed with primary achalasia and with no previous history of $\mathrm{PD}$, botulinum toxin injection, surgical myotomy or POEM at Samsung Medical Center, Seoul, Korea between January 2002 and December 2010. The diagnosis of achalasia was made based on the results of the radiographic, endoscopic and manometric studies according to accepted published criteria. ${ }^{1}$ All patients underwent PD as the initial treatment. The patients were divided into the 2 groups: (1) who developed GERD after PD (GERD group) and (2) who did not develop GERD after PD (non-GERD group) during follow-up. GERD was defined as pathologic acid exposure (PAE), reflux esophagitis or typical reflux symptoms. PAE was defined as an intra-esophageal $\mathrm{pH}$ of $<4$ for more than $4.0 \%$ of the recording time of 24-hour $\mathrm{pH}$ monitoring. Reflux esophagitis was defined by esophagogastroduodenoscopy (EGD). Typical reflux symptoms were defined as heartburn and/or acid regurgitation. Heartburn was described as "a burning sensation rising from the lower chest up toward the neck," and acid regurgitation was described as "regurgitation of acidic fluid from the stomach or lower chest to the throat." ${ }^{15}$ However, the patients with both reflux symptoms and dysphagia concurrently were not included into GERD group. The study was conducted according to the principles of the Declaration of Helsinki and approved by the Institutional Review Board at Samsung Medical Center, Seoul, Korea (No. SMC 2013-11-032).

\section{Initial Evaluation and Follow-up}

The pretreatment evaluation consisted of symptom assessment, EGD, esophageal manometry and 24-hour pH monitoring. Symptoms were scored using the Eckardt score, which is the sum of the scores for dysphagia, regurgitation and chest pain on a scale from 0 to 3 ( $0=$ absent, $1=$ occasional, $2=$ daily and $3=$ each meal $)$ and weight loss ( $0=$ no weight loss, $1=<5 \mathrm{~kg}, 2=$ $5-10 \mathrm{~kg}, 3=>10 \mathrm{~kg}$ ). The total score ranges from 0 to 12 points. Recurrence was defined as recurred or aggravated symptoms of achalasia requiring additional treatment together with compatible radiographic, endoscopic and manometric study results during follow-up.

Patients underwent EGD, esophageal manometry and 24-hour $\mathrm{pH}$ monitoring 1 month after the initial treatment and yearly thereafter and at the time of symptom recurrence.

\section{Esophageal Manometry}

Esophageal manometry was conducted with the patient in the supine position, using an eight-lumen polyvinyl manometric tube with 4 distal side holes and 4 proximal holes situated 5-cm apart (ESM38R; Armdorfer Medical Specialties, Greendale, WI, USA). The manometric tube was transnasally introduced, and then slowly withdrawn in 1-cm increments by station pull-through in order to measure the LES resting and residual pressures. The LES relaxation was evaluated with wet swallows of $5 \mathrm{~mL}$ of water. Completeness of relaxation was assessed via measurements of residual LES pressure as compared with resting LES pressure. Peristalsis was assessed by positioning at least three pressure sensors situated at 5 -cm intervals within the body of the esophagus. The distal sensor was positioned at a level 3-cm above 
the LES and a series of 10 wet swallows was conducted. From September 2008, esophageal manometry was conducted using the high-resolution manometry (HRM) system (Sandhill Scientific Inc., Highlands Ranch, CO, USA) in a standard manner. The HRM probe has 32 circumferential pressure sensors spaced $1 \mathrm{~cm}$ apart. The HRM probe was transnasally introduced and positioned with about 5 intragastric sensors.

\section{Ambulatory 24-hour Esophageal pH Monitoring}

Twenty-four hour $\mathrm{pH}$ monitoring was performed using a 2.1-mm monocrystalline $\mathrm{pH}$ catheter equipped with 2 antimony electrodes (Synectics, Irving, TX, USA). The $\mathrm{pH}$ catheters were calibrated at $37^{\circ} \mathrm{C}$ in standard buffer solution at $\mathrm{pHs}$ of 7 and 1 (Fisher Scientific, Fairlawn, NJ, USA), both before and after monitoring. The catheters were introduced transnasally, in order to position the sensors 5 -cm above the upper border of the manometrically determined LES. The $\mathrm{pH}$ electrodes were connected to a portable digital data recorder (Mark II Gold; Synectics), which stored $\mathrm{pH}$ data every 4 seconds, for up to 24 hours. Patients returned home with instructions to keep a diary recording symptoms, meal times, time to bed and waking time. Patients were encouraged to do normal daily activities with no dietary restrictions. Patients returned the next day (after 18-24 hours) to have the probes removed and the diaries reviewed. Esophageal acid exposure values (percentage of time $\mathrm{pH}<4$ ) were calculated with a commercial software program (EsoPHogram, version 5.70C2; Gastrosoft, Irving, TX, USA). From January 2006, for 24-hour $\mathrm{pH}$ monitoring, a portable data logger (Sandhill Scientific Inc.) connected to a single-use combined impedance and $\mathrm{pH}$ probe (Sandhill Scientific Inc.) was used. Data analysis was performed using the BioView MII software (Sandhill Scientific Inc.).

\section{Pneumatic Balloon Dilatation}

PD was performed under fluoroscopic guidance with the use of a Rigiflex dilator (Boston Scientific, Boston, MA, USA). All of the patients fasted overnight and received topical anesthesia for the pharynx and intravenous midazolam and/or pethidine. The balloon of the dilator was positioned at the gastroesophageal junction under the guidance of the fluoroscope. It was then inflated until a minimum pressure of 10 psi was achieved, with the waist remaining in a stable position. Dilatation was conducted with the aim of maintaining this pressure for 2 minutes and obliterating the waist of the balloon. Single PD was performed using a $3.0 \mathrm{~cm}$ balloon.

\section{Study Endpoint}

The primary endpoint was recurrence of achalasia during follow-up. We defined RFS as the time from the first PD to the date of symptom recurrence. For RFS, patients without recurrence were censored at the last follow-up visit. Early recurrence was defined as any recurrence that occurred within 3 years after the initial PD and late recurrence as 3 years or more.

\section{Statistical Methods}

Statistical analyses were conducted using PASW Statistics 18 for Windows (SPSS, Inc., Chicago, IL, USA). Shapiro-Wilk test was performed for normality. The statistical results are presented as mean $\pm \mathrm{SD}$, median (interquartile range) or number of patients (\%). Continuous variables were compared parametrically using Student's $t$ test or non-parametrically using the MannWhitney U test. Categorical variables were compared using the $\chi^{2}$ test or Fisher's exact test as appropriate. Wilcoxon's signed ranks test and Student's paired $t$ test were used to evaluate changes of LES pressure and LES relaxation after $\mathrm{PD}$, respectively. One-way ANOVA and Kruskal Wallis test were used to compare changes of LES pressure and LES relaxation after PD between the two groups. The drop of LES pressure was defined as the \% change (decreased) of LES pressure after PD. RFS was calculated using the Kaplan-Meier method and compared using the log-rank test. A two-sided $P$-value $<0.05$ was taken as statistically significant.

\section{Results}

\section{Patients}

Two patients who did not have follow-up and one patient who developed esophageal cancer during follow-up were excluded from this study. Finally, a total of 79 consecutive patients were included in the current study. 63 patients underwent conventional manometry, while 16 underwent $\mathrm{HRM}$ at the time of making a diagnosis. Of them, 36 patients (45.6\%) were male and the mean age was $44.2 \pm 15.8$ years. The median initial Eckardt score was 6 (4-9) and the median duration of symptoms before treatment was 3 months (2-9 months). Twenty-one patients (26.6\%) were diagnosed with GERD during follow-up after PD. Sixteen patients were diagnosed with GERD by PAE or reflux esophagitis while 5 were diagnosed with GERD by typical reflux 
Table 1. The Baseline Characteristics of Patients According to the Occurrence of Gastroesophageal Reflux Disease After Pneumatic Balloon Dilatation

\begin{tabular}{lccc}
\hline & GERD group $(\mathrm{n}=21)$ & Non-GERD group $(\mathrm{n}=58)$ & $P$-value \\
\hline Age $(\mathrm{yr})$ & $42.3 \pm 13.1$ & $44.8 \pm 16.8$ & 0.543 \\
Gender (male $[\%])$ & $13(61.9)$ & $23(39.7)$ & 0.079 \\
Body mass index $\left(\mathrm{kg} / \mathrm{m}^{2}\right)$ & $21.3(18.6-23.1)$ & $20.4(18.9-23.2)$ & 0.995 \\
Initial Eckardt symptom score & $6(6-9)$ & $6(3-9)$ & 0.591 \\
Duration of symptoms before treatment $(\mathrm{mo})$ & $5.0(2.0-8.5)$ & $3.0(1.0-9.0)$ & 0.396 \\
Pre-treatment LES pressure $(\mathrm{mmHg})$ & $41.7(24.4-51.5)$ & $39.7(29.2-50.4)$ & 0.845 \\
Pre-treatment LES relaxation $(\%)$ & $60.9 \pm 18.8$ & $71.2 \pm 19.7$ & 0.079 \\
\hline
\end{tabular}

LES, lower esophageal sphincter.

Data are presented as mean $\pm \mathrm{SD}$, median (interquartile range) or number of patients (\%).

Table 2. Post-treatment Lower Esophageal Sphincter Pressure Analysis

\begin{tabular}{lccc}
\hline & GERD group $(\mathrm{n}=20)$ & Non-GERD group $(\mathrm{n}=46)$ & $P$-value \\
\hline Post-treatment LES pressure $(\mathrm{mmHg})$ & $29.2(20.6-37.1)$ & $25.3(16.4-34.6)$ & 0.395 \\
Post-treatment LES relaxation (\%) & $84.2(75.1-98.0)$ & $84.2(76.0-95.8)$ & 0.987 \\
Decrease of LES pressure after PD (mmHg) & $8.9(-0.7-20.7)$ & $13.8(5.7-26.4)$ & 0.149 \\
Drop of LES pressure (\%) & $20.4(-2.8-66.5)$ & $42.4(14.5-56.3)$ & 0.370 \\
Increase of LES relaxation after PD (\%) & $20.7 \pm 20.8$ & $12.9 \pm 21.0$ & 0.239 \\
\hline
\end{tabular}

GERD, gastroesophageal reflux disease; LES, lower esophageal sphincter; PD, pneumatic balloon dilatation.

Data are presented as mean $\pm \mathrm{SD}$ or median (interquartile range).

symptoms. The median time of diagnosis of GERD was 8 months (2.0-20.4 months). Baseline characteristics of the 2 groups are shown in Table 1. There are no significant differences between the two groups regarding age, gender, body mass index, initial Eckardt score, duration of symptoms before treatment, pre-treatment LES pressure and pre-treatment LES relaxation.

\section{Treatment Short-term Outcomes}

All enrolled patients showed symptom improvement after PD. There was no perforation after PD. Post-treatment LES pressure was measured in 66/79 patients (20 in GERD and 46 in non-GERD group). The LES pressure decreased from 39.9 $\mathrm{mmHg}$ (28.7-50.3 $\mathrm{mmHg})$ to $28.1 \mathrm{mmHg}$ (17.6-34.9 $\mathrm{mmHg})$ after $\mathrm{PD}(P<0.001)$. The LES relaxation increased from $66.7 \pm 17.7 \%$ to $81.7 \pm 18.5 \%(P<0.001)$ after PD. Post-treatment LES pressure variables of the 2 groups are shown in Table 2. The change of LES pressure variables after PD was analyzed using available paired data. In GERD group, the LES pressure decreased from $41.7 \mathrm{mmHg}(24.4-51.5 \mathrm{mmHg})$ to 29.2 mmHg (20.8-37.8 mmHg) $(P=0.018$, Wilcoxon's signed ranks test with available data) and the LES relaxation increased from $62.6 \pm 17.4 \%$ to $83.5 \pm 16.5 \%(P=0.001$, Student's paired $t$ test with available data) after PD. In non-GERD group, the LES pressure decreased from $39.7 \mathrm{mmHg}$ (29.2-50.3 $\mathrm{mmHg})$ to $25.3 \mathrm{mmHg}(16.4-34.6 \mathrm{mmHg})(P<0.001$, Wilcoxon's signed ranks test with available data) and the LES relaxation increased from $68.3 \pm 17.7 \%$ to $81.0 \pm 19.4 \%(P=$ 0.001 , Student's paired $t$ test with available data) after PD. Decrease of LES pressure and increase of LES relaxation did not differ between the 2 groups (GERD group vs. non-GERD group; $8.9 \mathrm{mmHg}(-0.7-20.7 \mathrm{mmHg})$ vs. $13.8 \mathrm{mmHg}(5.7-26.4$ $\mathrm{mmHg}), P=0.149$, Kruskall Wallis test and $20.7 \pm 20.8$ vs. $12.9 \pm 21.0, P=0.239$, One-way ANOVA, respectively). The drop of LES pressure also did not differ between the 2 groups (GERD group vs. non- GERD group; $20.4 \%$ (-2.8-66.5\%) vs. $42.4 \%$ (14.5-56.3\%), $P=0.370$, Kruskall Wallis test).

\section{Treatment Long-term Outcomes}

During a median follow-up of 17.8 months (7.1-42.7 months), recurrence was occurred in $19.0 \%(n=15)$. There was no significant difference in terms of recurrence between the two groups by using the Kaplan-Meier method $(P=0.205$, log rank test; Figure). In addition, age, gender, body mass index, initial Eckardt score, duration of symptoms before treatment, pre-treat- 


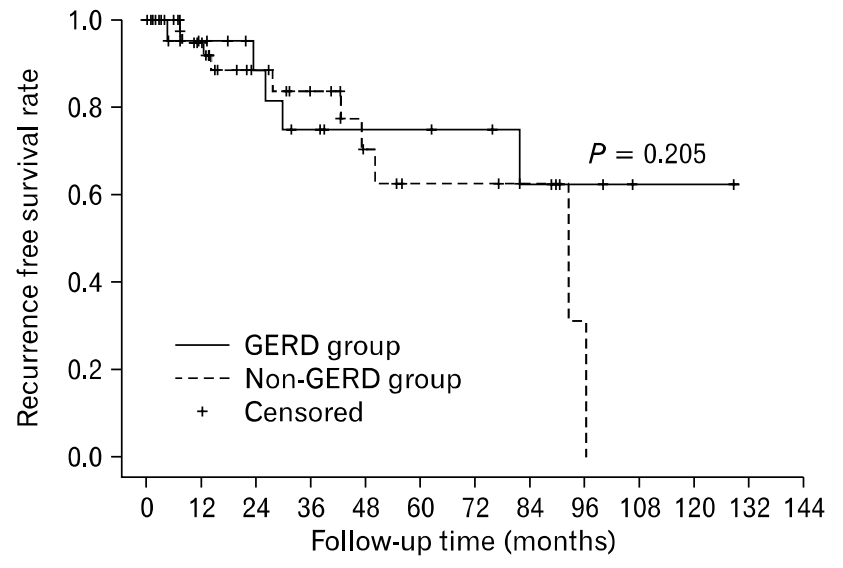

Figure. Kaplan-Meier curves showing the recurrence of achalasia after pneumatic dilatation in gastroesophageal reflux disease (GERD) group and non-GERD group.

ment LES pressure, pre-treatment LES relaxation, post-treatment LES pressure, post-treatment LES relaxation, decrease of LES pressure, drop of LES pressure and increase of LES relaxation were not associated with recurrence. Among the 15 patients with a recurrence of achalasia, 13 underwent second $\mathrm{PD}$. In case of early recurrence $(\mathrm{n}=8$, median 19.0 months, range 4.5-47.2 months), second PD was performed with a $3.5 \mathrm{~cm}$ balloon and the all patients showed symptom improvement after treatment. In case of late recurrence $(\mathrm{n}=5$, median 81.9 months, range 42.6-96.3 months) all patients also showed symptom improvement even after PD with a $3.0 \mathrm{~cm}$ balloon.

Among the 21 patients in GERD group, one patient was not followed after detection of GERD and 2 were not followed after starting proton pump inhibitor (PPI) use. In the remaining 18 patients, 14 had symptom relief on maintenance PPI therapy including on demand therapy $(n=1), 2$ were able to discontinue PPI therapy and 2 did not receive PPI therapy due to asymptomatic mild erosive esophagitis.

\section{Discussion}

Currently performed treatment modalities cannot cure achalasia. ${ }^{2,16}$ As such, each treatment aims to reduce the pressure gradient across the LES. ${ }^{1,8-10,17}$ Both PD and surgical myotomy are well-recognized modalities to disrupt LES for treatment in achalasia with comparable effectiveness. ${ }^{18,19}$ Recently developed POEM is also effective in lowering LES pressure, but requires longer follow-up and needs to be compared with PD or surgical myotomy. ${ }^{17,20-22}$ The most popular protocol of $\mathrm{PD}$ is a graded di- latation starting with a $3.0 \mathrm{~cm}$, followed by $3.5 \mathrm{~cm}$ and then 4.0 $\mathrm{cm}$ balloon, in subsequent sessions balloon. ${ }^{23}$ In our institution, a single dilatation with a $3.0 \mathrm{~cm}$ balloon is performed as the initial treatment and additional PD is performed according to an "on demand" strategy, based on symptom recurrence during follow-up. Even after a single PD, GERD often occurs. The occurrence of GERD is known to be associated with lower post-treatment LES pressure. ${ }^{24,25}$ We therefore hypothesized that GERD after PD could have a prognostic role for RFS in patients who received PD for achalasia. In addition, we investigated how often GERD occurs in achalasia patients who undergo PD as an initial treatment, what factors are associated with the occurrence of GERD and how the clinical course of patients with post-PD GERD is.

In the current study, 21 patients $(26.6 \%)$ were diagnosed with GERD after PD during follow-up. Between the GERD and non-GERD groups, there was no significant difference in demographic or clinical factors including pre- and post-treatment manometric results. Thus, a fourth of patients undergoing PD, even a single dilatation with a $3.0 \mathrm{~cm}$ balloon, are expected to experience GERD regardless of demographic or clinical factors. The incidence of GERD after PD has been reported to range from $4 \%$ to $35 \%{ }^{9,11,12,24,26}$ This wide range of incidence seems to stem from various different definitions used to make a diagnosis of GERD. In a prospective study by Novais and Lemme, ${ }^{24}$ they reported the incidence of gastroesophageal reflux (GER) of $31 \%$ using 24-hour $\mathrm{pH}$ tracing analysis to distinguish true GER patterns from other findings due to esophageal food fermentations. In the current study, there might be also little possibility that patients showing abnormal 24-hour $\mathrm{pH}$ monitoring findings due to food fermentation were erroneously included into GERD group. However, we did not include patients with concurrent dysphagia into GERD group and the patients suspicious of recurrence underwent other radiographic, endoscopic or manometric studies. Thus, we minimized the possibility of an erroneous inclusion of recurred patients into GERD group. Among the 12 patients who were diagnosed with GERD by PAE, 11 had available esophagographic data at the time of detection of PAE. Four patents had neither significant esophageal dilation nor passage disturbance on esophagography and 6 had improved and mild dilated $(<4 \mathrm{~cm})$ esophagus with mild passage disturbance. Remaining 1 patient had improved but moderately dilated esophagus $(4-6 \mathrm{~cm})$ together with PAE at the follow-up time of 1 month after PD. However, this patient did not have PAE before PD. Taken together, we believe that the incidence of GERD in 
the current study could reflect its true incidence.

On the contrary to our hypothesis, RFS of achalasia did not differ according to the occurrence of GERD. In patients who underwent PD, GERD occurring during follow-up is not a prognostic factor but a complication to be controlled. Although the time of diagnosis of GERD needs to be uniform to play a proper role in predicting outcomes, we could not do that in this retrospective study. Therefore, we performed additional analysis after excluding patients who developed GERD over 12 months after PD from GERD group. However, the incidence of recurrence did not differ consistently between the 2 groups even after the exclusion (data not shown). In the current study, all patients with GERD showed resolved or reduced reflux symptom with PPI therapy. This finding also supports that GERD detected in this study is from the true GER. To date, several studies have addressed predictors of outcome of PD. ${ }^{27}$ Age, gender, esophageal body diameter, balloon diameter, pre- and post-treatment LES pressure and timed barium esophagogram are factors useful to predict outcome of $\mathrm{PD}$, however these depend on the type of protocol and dilator used. ${ }^{28-33}$ Therefore our results also need to be interpreted in the context of a single PD protocol used.

We performed the first PD with a $3.0 \mathrm{~cm}$ balloon to avoid a procedure-related perforation, resulting in no perforation. There have been several reports showing a good long-term outcome of graded dilatation with progressively increasing balloon size. ${ }^{23,28,34}$ However, we did not routinely perform a subsequent PD with a larger balloon without an insufficient symptom relief. Instead, we performed the second PD in case of symptom recurrence requiring additional treatment with objective findings compatible with a recurrence. The size of balloon used was determined according to the time of recurrence. In case of early recurrence less than 3 years after the initial PD, a $3.5 \mathrm{~cm}$ balloon was used. However, in case of late recurrence, $\mathrm{PD}$ with a $3.0 \mathrm{~cm}$ balloon was repeated except one patient who recurred 47.2 months after the initial PD and received the second $\mathrm{PD}$ with a $3.5 \mathrm{~cm}$ balloon. All of the patients who underwent the second PD had satisfaction for symptom relief. Although the efficacy of PD strategy is out of the scope of this study, our data suggest that the "on demand" strategy after a single PD with a $3.0 \mathrm{~cm}$ balloon is effective and safe for treatment naïve patients with achalasia.

In our results, post-treatment LES pressure was measured in 66/79 patients (20 in GERD and 46 in non-GERD group). The median LES pressure significantly decreased from $39.9 \mathrm{mmHg}$ (28.7-50.3 $\mathrm{mmHg}$ ) to $28.1 \mathrm{mmHg}(17.6-34.9 \mathrm{mmHg})$ after PD. Ghoshal et al ${ }^{35}$ have reported $22.5 \mathrm{mmHg}$ as a best cut-off value of post-treatment LES pressure differentiating responders and non-responders after PD. However, among the current study patients, only 28 patients (43.9\%) showed post-treatment LES pressure within $22.5 \mathrm{mmHg}$ even though they all showed symptom improvement. This discrepancy might stem from the different definitions used in the each study. In the study by Ghoshal et al, ${ }^{35}$ response to PD was defined as a decrease in dysphagia score to 0 or 1 and/or total symptom score to $\leq 3$ on follow-up visit after PD. However, we evaluated the symptom response by subjective satisfaction for symptom relief. In addition, GERD occurred after PD in a significant number of patients and post-treatment LES pressure did not differ between the 2 groups. These observations suggest that the development of GERD in achalasia patients received PD is not associated with post-treatment LES pressure but rather associated with combined multiple factors which are affected by PD.

The current study had some limitations. First, the retrospective design may have introduced selection bias and underreporting of reflux symptoms. However, the presence or absence of reflux symptoms was well described in medical records. On the other hand, GERD could have been masked by PPI use for other symptoms. Among the 58 patients of non-GERD group, 5 took half dose PPI intermittently for their dyspeptic symptoms. There was no recurrence of achalasia in these patients. After excluding these 5 patients from the study, we re-analyzed the data but the results did not change regarding the comparison of RFS between the 2 groups (data not shown). Second, the study population was rather small and the follow-up duration was limited to draw firm conclusions. In the current study, to test the difference of RFS with a significant power between the 2 groups, several hundred patients were necessary with this follow-up time because there were many censored patients at an early follow-up time. This seems that some patients with improvement after treatment might not want to visit a clinic regularly further because there were also some patients who revisited clinic due to symptom recurrence after a certain period of follow-up loss. This made it difficult to conduct the current retrospective study with a good power. To overcome this limitation, a large prospective study with a strict real time data management is needed. Until then, however, our results would be of worth because this is the first study to determine whether GERD during follow-up after PD predicts the recurrence of achalasia. In addition, the current study includes data from 24-hour $\mathrm{pH}$ monitoring, which is considered the best diagnostic method for GER. In addition, we provided overall outcomes of a single PD with a $3.0 \mathrm{~cm}$ balloon with an "on demand" 
strategy. In conclusion, GERD occurs after even a single PD for achalasia in a significant number of patients. However, GERD after PD is well responsive to PPI therapy. Our data suggest that GERD during follow-up after PD does not have a prognostic role.

\section{References}

1. Spechler SJ, Castell DO. Classification of oesophageal motility abnormalities. Gut 2001;49:145-151.

2. Boeckxstaens GE, Zaninotto G, Richter JE. Achalasia. Lancet 2014; 383:83-93.

3. Vantrappen G, Hellemans J, Deloof W, Valembois P, Vandenbroucke J. Treatment of achalasia with pneumatic dilatations. Gut 1971;12:268-275.

4. Richter JE, Boeckxstaens GE. Management of achalasia: surgery or pneumatic dilation. Gut 2011;60:869-876.

5. Pasricha PJ, Ravich WJ, Hendrix TR, Sostre S, Jones B, Kalloo AN. Intrasphincteric botulinum toxin for the treatment of achalasia. N Engl J Med 1995;332:774-778.

6. Vaezi MF, Richter JE. Diagnosis and management of achalasia. American College of Gastroenterology Practice Parameter Committee. Am J Gastroenterol 1999;94:3406-3412.

7. Ali A, Pellegrini CA. Laparoscopic myotomy: technique and efficacy in treating achalasia. Gastrointest Endosc Clin N Am 2001;11:347358.

8. Vantrappen G, Hellemans J. Treatment of achalasia and related motor disorders. Gastroenterology 1980;79:144-154.

9. Richter JE. Modern management of achalasia. Curr Treat Options Gastroenterol 2005;8:275-283.

10. Boeckxstaens GE. Achalasia. Best Pract Res Clin Gastroenterol 2007;21:595-608.

11. Vela MF, Richter JE, Khandwala F, et al. The long-term efficacy of pneumatic dilatation and Heller myotomy for the treatment of achalasia. Clin Gastroenterol Hepatol 2006;4:580-587.

12. Dobrucali A, Erzin Y, Tuncer M, Dirican A. Long-term results of graded pneumatic dilatation under endoscopic guidance in patients with primary esophageal achalasia. World J Gastroenterol 2004;10: 3322-3327.

13. Abir F, Modlin I, Kidd M, Bell R. Surgical treatment of achalasia: current status and controversies. Dig Surg 2004;21:165-176.

14. Swanstrom LL, Kurian A, Dunst CM, Sharata A, Bhayani N, Rieder E. Long-term outcomes of an endoscopic myotomy for achalasia: the POEM procedure. Ann Surg 2012;256:659-667.

15. Fock KM, Talley NJ, Fass R, et al. Asia-Pacific consensus on the management of gastroesophageal reflux disease: update. J Gastroenterol Hepatol 2008;23:8-22.

16. Vaezi MF, Pandolfino JE, Vela MF. ACG clinical guideline: diagnosis and management of achalasia. Am J Gastroenterol 2013;108: 1238-1249.

17. Inoue $\mathrm{H}$, Minami $\mathrm{H}$, Kobayashi $\mathrm{Y}$, et al. Peroral endoscopic myotomy (POEM) for esophageal achalasia. Endoscopy 2010;42:265-271.

18. Boeckxstaens GE, Annese V, des Varannes SB, et al. Pneumatic dilation versus laparoscopic Heller's myotomy for idiopathic achalasia. $\mathrm{N}$
Engl J Med 2011;364:1807-1816.

19. Shimi S, Nathanson LK, Cuschieri A. Laparoscopic cardiomyotomy for achalasia. J R Coll Surg Edinb 1991;36:152-154.

20. von Renteln D, Inoue H, Minami H, et al. Peroral endoscopic myotomy for the treatment of achalasia: a prospective single center study. Am J Gastroenterol 2012;107:411-417.

21. Lee BH, Shim KY, Hong SJ, et al. Peroral endoscopic myotomy for treatment of achalasia: initial results of a korean study. Clin Endosc 2013;46:161-167.

22. von Renteln D, Fuchs KH, Fockens P, et al. Peroral endoscopic myotomy for the treatment of achalasia: an international prospective multicenter study. Gastroenterology 2013;145:309-311, e3.

23. Kadakia SC, Wong RK. Graded pneumatic dilation using Rigiflex achalasia dilators in patients with primary esophageal achalasia. Am J Gastroenterol 1993;88:34-38.

24. Novais PA, Lemme EM. 24-h pH monitoring patterns and clinical response after achalasia treatment with pneumatic dilation or laparoscopic Heller myotomy. Aliment Pharmacol Ther 2010;32:12571265.

25. Tuset JA, Luján M, Huguet JM, Canelles P, Medina E. Endoscopic pneumatic balloon dilation in primary achalasia: predictive factors, complications, and long-term follow-up. Dis Esophagus 2009;22:74-79.

26. Zerbib F, Thétiot V, Richy F, Benajah DA, Message L, Lamouliatte $\mathrm{H}$. Repeated pneumatic dilations as long-term maintenance therapy for esophageal achalasia. Am J Gastroenterol 2006;101:692-697.

27. Ghoshal UC, Rangan M. A review of factors predicting outcome of pneumatic dilation in patients with achalasia cardia. J Neurogastroenterol Motil 2011;17:9-13.

28. Ghoshal UC, Kumar S, Saraswat VA, Aggarwal R, Misra A, Choudhuri G. Long-term follow-up after pneumatic dilation for achalasia cardia: factors associated with treatment failure and recurrence. Am J Gastroenterol 2004;99:2304-2310.

29. Ponce J, Garrigues V, Pertejo V, Sala T, Berenguer J. Individual prediction of response to pneumatic dilation in patients with achalasia. Dig Dis Sci 1996;41:2135-2141.

30. Mehta R, John A, Sadasivan S, et al. Factors determining successful outcome following pneumatic balloon dilation in achalasia cardia. Indian J Gastroenterol 2005;24:243-245.

31. Eckardt VF, Aignherr C, Bernhard G. Predictors of outcome in patients with achalasia treated by pneumatic dilation. Gastroenterology 1992;103:1732-1738.

32. Vaezi MF, Baker ME, Achkar E, Richter JE. Timed barium oesophagram: better predictor of long term success after pneumatic dilation in achalasia than symptom assessment. Gut 2002;50:765-770.

33. Chuah SK, Hu TH, Wu KL, et al. Clinical remission in endoscope-guided pneumatic dilation for the treatment of esophageal achalasia: 7-year follow-up results of a prospective investigation. J Gastrointest Surg 2009;13:862-867.

34. Farhoomand K, Connor JT, Richter JE, Achkar E, Vaezi MF. Predictors of outcome of pneumatic dilation in achalasia. Clin Gastroenterol Hepatol 2004;2:389-394.

35. Ghoshal UC, Rangan M, Misra A. Pneumatic dilation for achalasia cardia: reduction in lower esophageal sphincter pressure in assessing response and factors associated with recurrence during long-term follow up. Dig Endosc 2012;24:7-15. 